\title{
Polarization Properties of Quasi-Homogeneous Beams Propagating in Oceanic Turbulence
}

\author{
Feinan Chen*, Qi Zhao, Yanru Chen, and Jingjing Chen \\ Department of Optical Engineering and Electronic Technology, NJUST, NanJing 210094, \\ JiangSu Province, China
}

(Received February 25, 2013 : revised March 25, 2013 : accepted April 2, 2013)

\begin{abstract}
Based on the extended Huygens-Fresnel principle and generalized Stokes theory, the evolution of polarization properties of beams generated by quasi-homogenous $(\mathrm{QH})$ sources propagating in clear oceanic water was studied by the use of the oceanic turbulence spatial spectrum function. The results show that the beams have similar polarization self-reconstructed behavior under different turbulence conditions in the far field, but if the propagation distance is not long enough, the degree of polarization (DOP) fluctuates with much more complexity than state of polarization (SOP) of $\mathrm{QH}$ beams. The self-reconstructed ability of DOP at the special distance in turbulence would get to the best value if the values of coherence of width were chosen suitably, but for SOP, it has no best value.
\end{abstract}

Keywords: Quasi-Homogenous source, Oceanic turbulence, Polarization self reconstruction

OCIS codes : (010.4450) Oceanic optics; (010.4455) Oceanic propagation; (030.7060) Turbulence; (260.5430) Polarization

\section{INTRODUCTION}

The QH beams which can be generated by SLMs have been studied and used widely in recent years [1]. The coherence and polarization properties of quasi-homogenous sources in the far field has been studied by Korotokva [2]. The coherence of properties of the field generated by a beam radiated from a quasi-homogenous electromagnetic source scattering on $\mathrm{QH}$ media has been studied by Xin [3]. Chen and Li have been researched the coherence properties and polarization modulation of $\mathrm{QH}$ beams scattered from anisotropic media [4-5]. The propagation properties of laser beams in random media like turbulence or human tissue are important for applications such as optical imaging, remote sensing and communication systems so more and more researchers are attracted [6-11]. The polarization "self-reconstructed" phenomenon in atmosphere turbulence has been known for a long time. The normalized spectrum of the beam generated by $\mathrm{QH}$ source after propagating through turbulent media has been proved to be equal to the normalized spectrum of the source [12]. The degree of polarization of electromagnetic Gaussian-Schell beams and partially coherent electromagnetic flat-topped beams also have self-reconstructed properties in atmospheric turbulence [13-14]. However, recent reports have shown that beams generated from anisotropic sources don't have polarization self-reconstructed properties in turbulent media [15-16]. On the other hand, oceanic turbulence is an important natural random medium but the light propagation though oceanic turbulence is a relatively unexplored topic compared to that in other media. The spatial spectrum model of oceanic turbulence combining temperature and salinity fluctuations is only used by a few articles to study the light propagation in the oceanic waters [16-18].

In this paper, we focused on the polarization features of $\mathrm{QH}$ beams propagating through oceanic turbulence. The analytical expressions of on-axis generalized Stokes parameters of QH beams were calculated, then the variations of DOP and SOP of QH beams traveling through oceanic turbulence have been simulated. The self-reconstructed properties of such beams in the far field in turbulent media have been analyzed under different source parameters and turbulence conditions.

\footnotetext{
*Corresponding author: feinanchen617@163.com

Color versions of one or more of the figures in this paper are available online.
} 


\section{METHODS}

For beams generated by quasi-homogenous $(\mathrm{QH})$ sources the spectral density $S^{(0)}(\mathbf{r})$ varies much more slowly with position vector than the correlation coefficients $\mu_{i j}{ }^{(0)}(\mathbf{r}, \omega)$ change with difference of position vector $\mathbf{r}_{1}-\mathbf{r}_{2}$. The cross spectral density matrix of $\mathrm{QH}$ sources can be expressed as:

$$
\overline{\mathbf{W}}^{(0)}=W_{i j}^{(0)}\left(\mathbf{r}, \mathbf{r}_{2}, \omega\right)=\alpha_{i j}(\omega) S^{(0)}\left[\left(\mathbf{r}_{1}+\mathbf{r}_{2}\right) / 2, \omega\right] \mu_{i j}^{(0)}\left(\mathbf{r}_{1}-\mathbf{r}_{2}, \omega\right)
$$

The superscript (0) denotes quantity pertaining to the incident field. The spectral density can be expressed as the trace of cross spectral density matrix: $S^{(0)}(\mathbf{r}, \omega)=\operatorname{Tr} \overleftrightarrow{W}^{(0)}$ $\left(\mathbf{r}_{1}, \mathbf{r}_{2}, \omega\right), a_{i j}(\omega)$ is the frequency dependent factor which connects the spectral components: $S_{y}^{(0)}(\omega)=\alpha(\omega) S_{x}^{(0)}(\omega)$.

$$
\alpha_{i j}(\omega)= \begin{cases}\frac{1}{1+\alpha(\omega)} & \text { when } i=j=x \\ \frac{\alpha(\omega)}{1+\alpha(\omega)} & \text { when } i=j=y \\ \frac{\sqrt{\alpha(\omega)}}{1+\alpha(\omega)} & \text { when } i \neq j\end{cases}
$$

For $\alpha=0$, the source generated linearly polarized light in the $\mathrm{x}$ direction, for $\alpha \rightarrow \infty$, the light is linearly polarized in the y direction. Without loss of generality, the spectral density and correlation coefficients can be described by the Gaussian-Schell model:

$$
\begin{aligned}
& S^{(0)}(\mathbf{r}, \omega)=A \exp \left(-\mathbf{r}^{2} / 2 \sigma^{2}\right) \\
& \mu\left(\mathbf{r}_{1}, \mathbf{r}_{2}, \omega\right)=B_{i j} \exp \left[\frac{\left(\mathbf{r}_{1}-\mathbf{r}_{2}\right)^{2}}{2 \delta_{i j}^{2}}\right]
\end{aligned}
$$

$A$ is spectral amplitude which we set $A=1$ in this article, and $B_{i j}$ is called the coefficient of correlation between the incident field components, for quasi-homogenous beams, it can be set as: $B_{x x}=B_{y y}=1, B_{x y}=B_{y x}^{*}$, where the asterisk means complex conjugate. $\sigma$ represents the width of the source, $\delta_{i j}$ represents the coherent width of the source. Several conditions of the source must be satisfied in order to produce a physically reliable $\mathrm{QH}$ beam [19].

The cross spectral density matrix of the beam at the receiving plane can be derived by the extended HuygensFresnel principle:

$$
\begin{aligned}
& W_{i j}\left(\boldsymbol{\rho}_{1}, \boldsymbol{\rho}_{2}, z ; \omega\right)=\frac{k^{2}}{4 \pi^{2} z^{2}} \iint d^{2} r_{1} \iint d^{2} r W_{i j}^{(0)}(\mathbf{r}, \mathbf{r}, \omega) \exp \left[-i k \frac{\left(\boldsymbol{\rho}_{1}-\mathbf{r}_{1}\right)^{2}-\left(\boldsymbol{\rho}_{2}-\mathbf{r}_{2}\right)^{2}}{2 z}\right] \\
& \left.\left\langle\exp \left[\psi^{\prime \prime}\left(\mathbf{\rho}, \mathbf{r}_{1}\right)+\psi\left(\mathbf{p}_{2}, \mathbf{r}\right)\right]\right\rangle\right]
\end{aligned}
$$

Where $\mathbf{r}_{1}, \mathbf{r}_{2}$ are two arbitrary position vectors of points on the source plane while $\rho_{1}, \rho_{2}$ are two position vectors of observation points on the receiving plane. $z$ is propagation distance and $k=\frac{2 \pi}{\lambda}$ is the wave number of the beam. The last term in Eq. (5) represents the correlation function of the complex phase perturbed by random media :

$$
\begin{aligned}
& \left\langle\exp \left[\psi^{*}\left(\boldsymbol{\rho}_{1}, \mathbf{r}_{1}\right)+\psi\left(\boldsymbol{\rho}_{2}, \mathbf{r}_{2}\right)\right]\right\rangle= \\
& \exp \left\{-\frac{1}{3} \pi^{2} k^{2} z T\left[\left(\boldsymbol{\rho}_{1}-\boldsymbol{\rho}_{2}\right)^{2}+\left(\mathbf{r}_{1}-\mathbf{r}_{2}\right)\left(\boldsymbol{\rho}_{1}-\mathbf{\rho}_{2}\right)+\left(\mathbf{r}_{1}-\mathbf{r}_{2}\right)^{2}\right]\right\}
\end{aligned}
$$

Where $T=\int_{0}^{\infty} \kappa^{3} \Phi(\kappa) d \kappa$ describes the strength of turbulence perturbation.

The model of the spatial power spectrum of refractive index fluctuations of homogenous and isotropic oceanic turbulence can be described as a linearly polynomial of temperature fluctuations and salinity fluctuations:

$$
\begin{aligned}
& \Phi_{n}(\kappa)=0.388 \times 10^{-8} \xi^{-1 / 3} \kappa^{-11 / 3}\left[1+2.35(\kappa \eta)^{2 / 3}\right] \\
& \times \frac{\chi_{T}}{w^{2}}\left(\mathrm{w}^{2} e^{-A_{T} \delta}+e^{-A_{s} \delta}-2 \mathrm{w} e^{-A_{A_{S}} \delta}\right)
\end{aligned}
$$

In Eq. (7), $\xi$ is the rate of dissipation of kinetic energy per unit mass of fluid, ranging from $10^{-4} \mathrm{~m}^{2} \mathrm{~s}^{-3}$ in turbulence surface layer to $10^{-10} \mathrm{~m}^{2} \mathrm{~s}^{-3}$ in the mid-water column. $\chi_{T}$ is the rate of dissipation of mean squared temperature ranges from $10^{-2} K^{2} s^{-1}$ below the oceanic turbulence to $10^{-10} K^{2} s^{-1}$ at the mid-water column. $\eta$ is the Kolmogorov micro scale. Other parameters in Eq. (7) are:

$$
A_{T}=1.863 \times 10^{-2}, \quad A_{S}=1.9 \times 10^{-4}, \quad A_{T S}=9.41 \times 10^{-3}, \quad \delta=8.284(\kappa \eta)^{4 / 3}
$$

$+12.978(\mathrm{k} \eta)^{2}$; w defines the ratio of temperature to salinity contributions to refractive index spectrum which in oceanic waters varies in the interval $[-5 ; 0]$, with -5 and 0 corresponding to dominating temperature-induced and salinityinduced optical turbulence, respectively.

From Eq. (3)-Eq. (6), the cross spectral density matrix of the electromagnetic field on the receiving plane can be expressed as:

$$
\begin{aligned}
& W_{i j}\left(\mathbf{\rho}_{1}, \boldsymbol{\rho}_{2}, z ; \omega\right)=\frac{k^{2}}{4 \pi^{2} z^{2}} \iint d^{2} \mathbf{r}_{1} \iint d^{2} \mathbf{r}_{2} \alpha_{i j} A B_{i j} S^{(0)}\left[\left(\mathbf{r}_{1}+\mathbf{r}_{2}\right) / 2, \omega\right] \mu_{i j}^{(0)}\left(\mathbf{r}_{1}-\mathbf{r}_{2}, \omega\right) \\
& \exp \left\{-\frac{\pi^{2} k^{2} z}{3} T\left[\left(\boldsymbol{\rho}_{1}-\mathbf{\rho}_{2}\right)^{2}+\left(\mathbf{r}_{1}-\mathbf{r}_{2}\right)\left(\boldsymbol{\rho}_{1}-\boldsymbol{\rho}_{2}\right)+\left(\mathbf{r}_{1}-\mathbf{r}_{2}\right)^{2}\right]\right\} \\
& \exp \left[-i k \frac{\left(\boldsymbol{\rho}_{1}-\mathbf{r}_{1}\right)^{2}-\left(\boldsymbol{\rho}_{2}-\mathbf{r}_{2}\right)^{2}}{2 z}\right]
\end{aligned}
$$

As a matter of convenience, we make changes of the spatial arguments:

$$
\begin{array}{ll}
\mathbf{r}^{+}=\left(\mathbf{r}_{1}+\mathbf{r}_{2}\right) / 2 & \boldsymbol{\rho}^{+}=\left(\boldsymbol{\rho}_{1}+\boldsymbol{\rho}_{2}\right) / 2 \\
\mathbf{r}^{-}=\mathbf{r}_{1}-\mathbf{r}_{2} & \boldsymbol{\rho}^{-}=\boldsymbol{\rho}_{1}-\boldsymbol{\rho}_{2}
\end{array}
$$


With the help of Eq. (9), the cross spectral density matrix on the receiving plane is derived:

$$
\begin{aligned}
& W_{i j}\left(\boldsymbol{\rho}_{1}, \boldsymbol{\rho}_{2}, z ; \omega\right)=\frac{k^{2} \sigma^{2} \alpha_{i j} A B_{i j}}{2 z^{2} M_{i j}} \exp \left[\left(\frac{N}{M_{i j}}-1\right) \frac{k i}{z} \boldsymbol{\rho}^{-} \boldsymbol{\rho}^{+}\right] \\
& \exp \left\{\left[\frac{N^{2}}{M_{i j}}-\frac{k^{2} \sigma^{2}}{2 z^{2}}-\frac{\pi^{2} k^{2} z}{3} T\right]\left(\boldsymbol{\rho}^{-}\right)^{2}-\frac{k^{2}}{4 z^{2} M_{i j}}\left(\mathbf{\rho}^{+}\right)^{2}\right\}
\end{aligned} ;
$$

Where

$$
\begin{aligned}
& M_{i j}=\frac{1}{2 \delta_{i j}^{2}}+\frac{\pi^{2} k^{2} z}{3} T+\frac{k^{2} \sigma^{2}}{2 z^{2}} ; \\
& N=\left(\frac{k^{2} \sigma^{2}}{2 z^{2}}-\frac{\pi^{2} k^{2} z T}{6}\right) ;
\end{aligned}
$$

The polarization properties of an electromagnetic beam at a point in space can be determined by use of Stokes parameters which have recently been generalized from one-point quantities to two-point counterparts [20]. They contain not only polarization properties but also coherence properties of beams [21] and can be measured by Young's interference experiment [22-23]. The changes of generalized Stokes parameters in optical system or random media are also interesting problems and have received a lot of attention [24-25]. The four parameters can be expressed as follows:

$$
S_{i}\left(\boldsymbol{\rho}_{1}, \boldsymbol{\rho}_{2}, \omega\right)=\operatorname{Tr}\left[\vec{W}\left(\boldsymbol{\rho}_{1}, \boldsymbol{\rho}_{2}, \omega\right) * \vec{\sigma}_{i}\right](i=0,1,2,3)
$$

where $\overleftrightarrow{\sigma}_{i}$ are Pauli spin matrices:

$$
\sigma_{0}=\left(\begin{array}{ll}
1 & 0 \\
0 & 1
\end{array}\right), \sigma_{1}=\left(\begin{array}{cc}
1 & 0 \\
0 & -1
\end{array}\right), \sigma_{2}=\left(\begin{array}{ll}
0 & 1 \\
1 & 0
\end{array}\right), \sigma_{3}=\left(\begin{array}{cc}
0 & i \\
-i & 0
\end{array}\right) .
$$

$\overleftrightarrow{W}\left(\rho_{1}, \rho_{2}, \omega\right)$ represents the cross-spectral density matrix (CSDM) of the beam:

$$
\vec{W}\left(\boldsymbol{\rho}_{1}, \boldsymbol{\rho}_{2}, \omega\right)=\left[\begin{array}{ll}
W_{x x}\left(\boldsymbol{\rho}_{1}, \boldsymbol{\rho}_{2}, \omega\right) & W_{x y}\left(\boldsymbol{\rho}_{1}, \boldsymbol{\rho}_{2}, \omega\right) \\
W_{y x}\left(\boldsymbol{\rho}_{1}, \boldsymbol{\rho}_{2}, \omega\right) & W_{y y}\left(\boldsymbol{\rho}_{1}, \boldsymbol{\rho}_{2}, \omega\right)
\end{array}\right]
$$

If we only considered polarization properties of one point, i.e., $\boldsymbol{\rho}_{1}=\boldsymbol{\rho}_{2}=\boldsymbol{\rho}$, From Eq. (10), each element of the CSDM can be derived as the expression of:

$$
W_{i j}(\mathbf{\rho}, z, \omega)=\frac{k^{2} \sigma^{2} a_{i j} A B_{i j}}{2 z^{2} M_{i j}} \exp \left\{-\frac{k^{2}}{4 z^{2} M_{i j}} \mathbf{\rho}^{2}\right\}
$$

Inserting Eq. (11) into Eq. (12), and using the conditions of $M_{x y}=M_{y x}, \quad \alpha_{x y}=\alpha_{y x}$ the analytical expressions of four Stokes parameters can be obtained:

$$
\begin{aligned}
& S_{0}(\boldsymbol{\rho}, z, \omega)=\frac{A k^{2} \sigma^{2}}{2 z^{2}}\left[\frac{a_{x x}}{M_{x x}} \exp \left(-\frac{k^{2}}{4 z^{2} M_{x x}} \boldsymbol{\rho}^{2}\right)+\frac{a_{y y}}{M_{y y}} \exp \left(-\frac{k^{2}}{4 z^{2} M_{y y}} \boldsymbol{\rho}^{2}\right)\right] \\
& S_{1}(\boldsymbol{\rho}, z, \omega)=\frac{A k^{2} \sigma^{2}}{2 z^{2}}\left[\frac{a_{x x}}{M_{x x}} \exp \left(-\frac{k^{2}}{4 z^{2} M_{x x}} \boldsymbol{\rho}^{2}\right)-\frac{a_{y y}}{M_{y y}} \exp \left(-\frac{k^{2}}{4 z^{2} M_{y y}} \boldsymbol{\rho}^{2}\right)\right] \\
& S_{2}(\boldsymbol{\rho}, z, \omega)=\frac{A k^{2} \sigma^{2} a_{x y}}{2 M_{x y} z^{2}}\left[\exp \left(-\frac{k^{2}}{4 z^{2} M_{x y}} \boldsymbol{\rho}^{2}\right)\left(B_{x y}+B_{y x}\right)\right] \\
& S_{3}(\boldsymbol{\rho}, z, \omega)=\frac{-i^{*} A k^{2} \sigma^{2} a_{y y}}{2 M_{y z} z^{2}}\left[\exp \left(-\frac{k^{2}}{4 z^{2} M_{y y}} \boldsymbol{\rho}^{2}\right)\left(B_{x y}-B_{y x}\right)\right]
\end{aligned}
$$

The polarization properties can be expressed as the functions of these four parameters.

We considered the degree of polarization and the state of polarization of $\mathrm{QH}$ beams in this paper. $\mathrm{DOP}$ is used to describe the polarized portion of a beam. $\mathrm{DOP}=1$ means perfectly polarized beam while $\mathrm{DOP}=0$ means unpolarized beam. The state of polarization (SOP) includes the azimuth angle and ellipticity. The azimuth angle $\theta$ is defined by the smallest angle formed by the positive $\mathrm{x}$-direction and the direction of major semi-axis of the ellipse. $\varepsilon$, which determines the shape of the ellipse, is the ratio of the major semi-axis and the minor semi-axis.

For convenient, we normalized three Stokes parameters by $S_{0}$ as:

$$
S_{1}=\frac{S_{1}}{S_{0}} ; s_{2}=\frac{S_{2}}{S_{0}} ; s_{3}=\frac{S_{3}}{S_{0}}
$$

The DOP and SOP can be expressed as the function of normalized Stokes parameters:

$$
\begin{aligned}
& P(\mathbf{\rho}, z, \omega)=\sqrt{s_{1}^{2}+s_{2}^{2}+s_{3}^{2}} \\
& \theta(\boldsymbol{\rho}, z, \omega)=\frac{1}{2} \arctan \left[\frac{s_{2}}{s_{1}}\right] \\
& \varepsilon(\boldsymbol{\rho}, z, \omega)=\frac{1}{2} \arcsin \left[\frac{s_{3}}{\sqrt{s_{1}^{2}+s_{2}^{2}+s_{3}^{2}}}\right]
\end{aligned}
$$

Because of the uniform polarization properties of quasihomogenous beams, we only need to consider the on-axis point of the beam. And in order to prove the polarization self-reconstructed properties of QH beam, we should study far-field polarization properties of normalized Stokes parameters. First, let's consider the limit as follow:

$$
F=\lim _{z \rightarrow \infty} \frac{s_{i}(0, z, \omega)}{S_{i}(0,0, \omega)}
$$

Where $s_{i}(0, z, \omega)$ are normalized Stokes parameters on the receiving plane while $s_{i}(0,0, \omega)$ are such parameters on the source plane. From Eq. (13), $s_{1}(0, z, \omega)=\frac{M_{y y} \alpha_{x x}-M_{x x} \alpha_{y y}}{M_{y y} \alpha_{x x}+M_{x x} \alpha_{y y}}$, 
and $s_{1}(0,0, \omega)=\frac{\alpha_{x x}-\alpha_{y y}}{\alpha_{x x}+\alpha_{y y}}$ can be obtained from Eq. (1) Eq. (4). Then substituting into the limit of the ratio of two parameters, we get:

$$
F=\lim _{z \rightarrow \infty} \frac{s_{1}(0, z, \omega)}{s_{1}(0,0, \omega)}=\lim _{z \rightarrow \infty} \frac{\left(\delta_{y y}^{2} \alpha_{x x}-\delta_{x x}^{2} \alpha_{y y}\right)\left(\alpha_{x x}+\alpha_{y y}\right)+\frac{\pi^{2} k^{2} z}{3} T}{\left(\delta_{y y}^{2} \alpha_{x x}+\delta_{x x}^{2} \alpha_{y y}\right)\left(\alpha_{x x}-\alpha_{y y}\right)+\frac{\pi^{2} k^{2} z}{3} T}
$$

In turbulent medium, $\left(\delta_{y y}^{2} \alpha_{x x} \mp \delta_{x x}^{2} \alpha_{y y}\right)\left(\alpha_{x x} \pm \alpha_{y y}\right) \ll \frac{\pi^{2} k^{2} z}{3} T$, so $F=1$, then we proved the self-reconstructed property of $s_{1}$. Other parameters in Eq. (14) can also be proved similarly, then DOP and SOP self-reconstructed properties can be derived directly. We set follow parameters to judge the ability of the self -reconstructed property of $\mathrm{QH}$ beams:

$P_{d}=|(P(z)-P(0)) / P(0)|, \theta_{d}=|(\theta(z)-\theta(0)) / \theta(0)|, \varepsilon_{d}=|(\varepsilon(z)-\varepsilon(0)) / \varepsilon(0)|$.

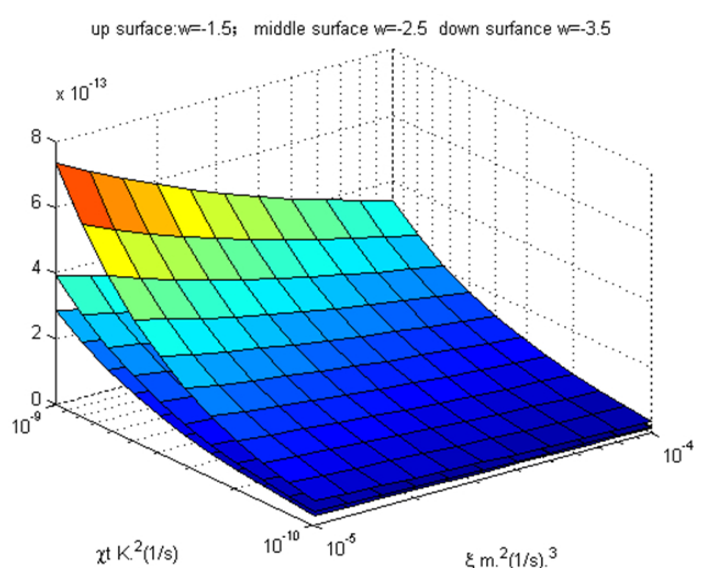

(a)
These parameters are modulus values of relative difference of the on-axis DOP and SOP between the source plane and the receiving plane at a special distance $\mathrm{z}$ in turbulences, the self-reconstructed ability will be better when these values get closer to 0 .

\section{RESULTS}

Figure 1 shows the strength of oceanic turbulence changes with turbulence parameters: w, $\chi_{T}$ and $\xi$. It can be easily found out that the strength of turbulence $\mathrm{T}$ increases with the increase of $\chi_{T}$ and $\mathrm{w}$, but reduces with the increases of $\xi$.

Figure 2 shows the changes of normalized Stokes parameters with distance at different $a$. The DOP and SOP we calculated in Eq. (17) and Eq. (19) could be expressed as the function of these parameters, so the behavior of generalized Stokes parameters decides the evolution of DOP and SOP of the beam through propagation.

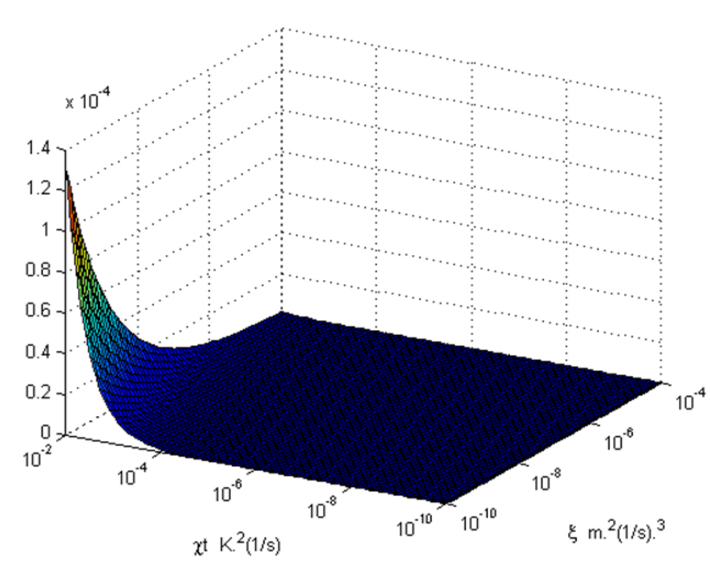

(b)

FIG. 1. Variations of turbulence strength due to temperature and salinity fluctuations

(The parameters in Fig. 1 are : (a) $\chi_{T}=10^{-9} K^{2}(1 / s) \sim 10^{-10} K^{2}(1 / s), \xi=10^{-4} m^{2}(1 / \mathrm{s})^{3} \sim, 10^{-5} m^{2}(1 / \mathrm{s})^{3}, \mathrm{w}=1.5,-2.5,-3.5,(\mathrm{~b}) \chi_{T}=10^{-2}$ $K^{2}(1 / \mathrm{s}) \sim 10^{-10} K^{2}(1 / \mathrm{s}), \xi=10^{-4} \mathrm{~m}^{2}(1 / \mathrm{s})^{3} \sim, 10^{-10} \mathrm{~m}^{2}(1 / \mathrm{s})^{3}, \mathrm{w}=1.5$.)

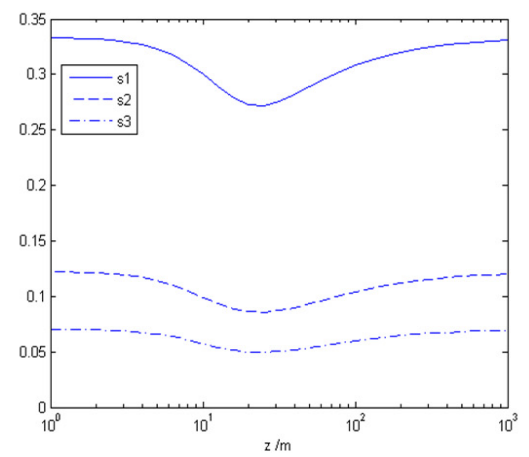

(a)

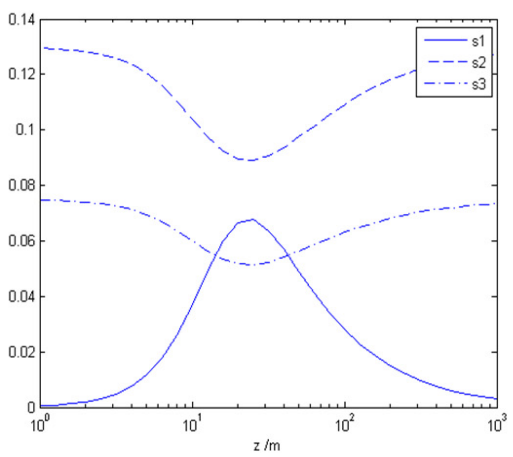

(b)

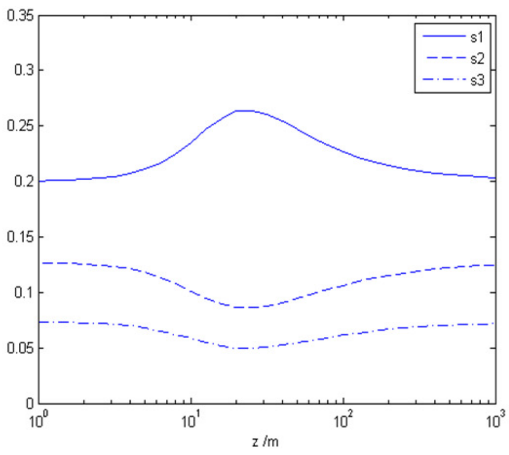

(c)

FIG. 2. Variations of on-axis generalized normalized Stokes parameters versus propagation distance $\mathrm{z}$ at different $\alpha$ : (a) $\alpha=0.5$, (b) $\alpha=1$, (c) $\alpha=1.5$. Other parameters are $\lambda=0.6328 \mu \mathrm{m}, \sigma=1 \mathrm{~cm}, B_{x y}=0.25 \exp (\mathrm{i} \pi / 6), B_{y x}=0.25 \exp (-\mathrm{i} \pi / 6), \quad \delta_{y y}=2 \delta_{x y} \delta_{x x}=1.5 \delta_{x y}$, $\delta_{x y}=0.2 \mathrm{~mm}$. 
In Fig. 3, we illustrated how the DOP and SOP of the $\mathrm{QH}$ beams depend on the parameters of oceanic turbulence. The effect of $\mathrm{w}$ on the changes of DOP and SOP has been shown in Fig. 3(a1)-(a3) and the effect of $\chi_{T}$ and $\xi$ has been shown in Fig. 3(b1)-(b3).

In Fig. 4, the influence of coherent width and the coefficients $\alpha_{i j}(\omega)$ on the changes of self-reconstructed properties of DOP of $\mathrm{QH}$ beams were revealed. While $\delta_{y y}$ and $\delta_{x y}$ are kept fixed, $c_{0}$ is the coefficient to decide the value of $\delta_{x x}$ as the form $\delta_{x x}=c_{0} \delta_{x y}$.

In Fig. 5, the self-reconstructed behavior of azimuth angle $\theta$ and the degree of ellipticity $\varepsilon$ were judged as the same method of DOP we plotted their variation versus $c_{0}$.

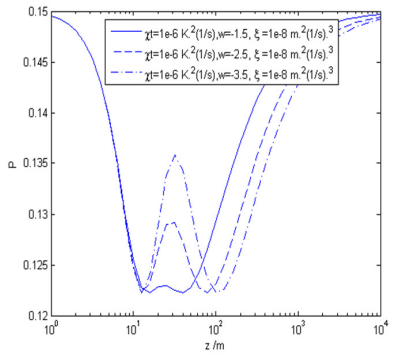

(a1)

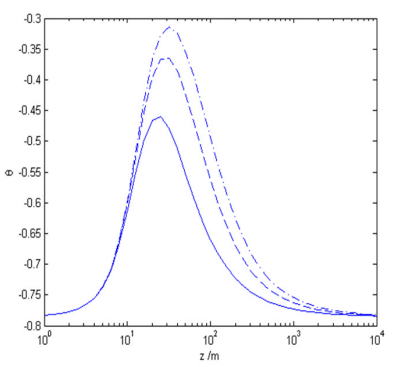

(b1)

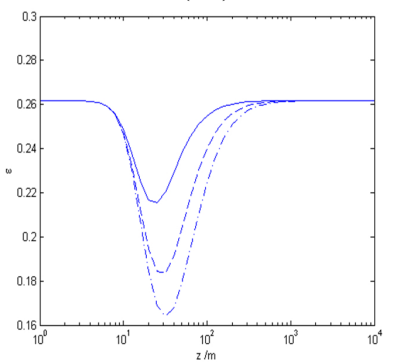

(c1)

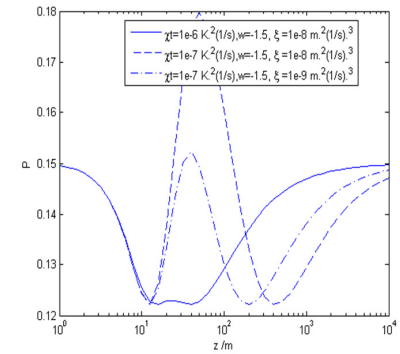

(a2)

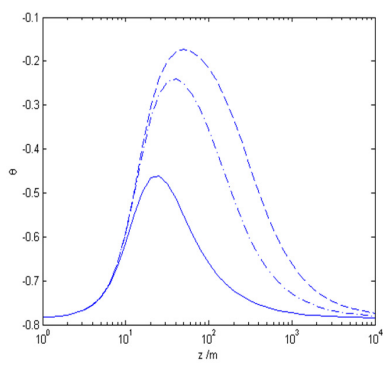

(b2)

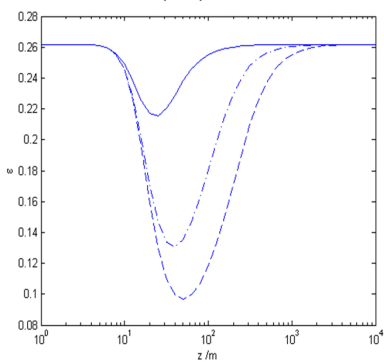

(c2)
FIG. 3. Variations of the on-axis DOP and SOP versus propagation distance $\mathrm{z}$ under different turbulence conditions. $\mathrm{a} 1, \mathrm{~b} 1$ are the changes of DOP, b1, b2 are the changes of $\theta$, $\mathrm{c} 1, \mathrm{c} 2$ are the changes of $\varepsilon$. We set $\alpha=1$, and other parameters are same with Fig. 2. The parameters of oceanic turbulence are set as (a1, b1, c1) $\chi_{t}=10^{-6} \mathrm{~K}^{2}(1 / \mathrm{s}), \quad \xi=10^{-8} \mathrm{~m}^{2}(1 / \mathrm{s})^{3}$. solid curve: $\mathrm{w}=-1.5$, dashed curve: $\mathrm{w}=-2.5$, dot-dashed curve: $\mathrm{w}=-3.5$. $(\mathrm{a} 2$, b2, c2) w=-1.5. solid curve: $\chi_{t}=10^{-6} K^{2}(1 / s), \quad \xi=10^{-8} \mathrm{~m}^{2}(1 / \mathrm{s})^{3}$, dashed curve: $\chi_{t}=10^{-7} \mathrm{~K}^{2}(1 / \mathrm{s}), \quad \xi=10^{-8} \mathrm{~m}^{2}(1 / \mathrm{s})^{3}$, dot-dashed curve: $\chi_{t}=10^{-7} K^{2}(1 / s), \quad \xi=10^{-9} \mathrm{~m}^{2}(1 / \mathrm{s})^{3}$.

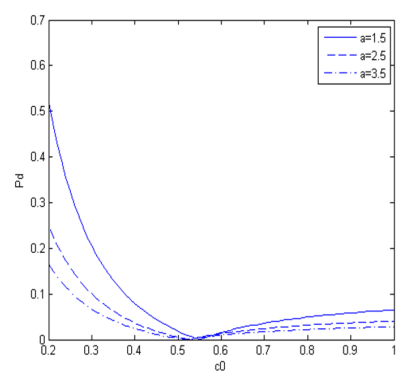

(a)

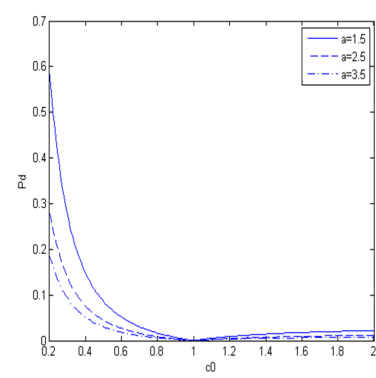

(b)
FIG. 4. Variations of $P_{d}$ with $c_{0}$ on the receiving plane at the propagation distance $\mathrm{z}=10 \mathrm{~km}$ under different $\alpha . c_{0}$ is the ratio of $\delta_{x x}$ to $\delta_{x y}$. Other parameters are same with Fig. 2. $\delta_{x y}=0.2 \mathrm{~mm}, \delta_{x x}=c_{0} \delta_{x y}$, (a) $\sigma=1 \mathrm{~cm}, \delta_{y y}=0.5 \delta_{x y}$, (b) $\sigma=1 \mathrm{~cm}$, $\delta_{y y}=\delta_{x y}$.

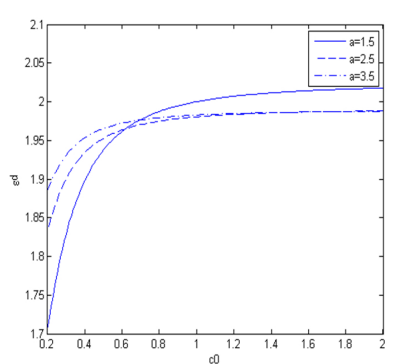

(a)

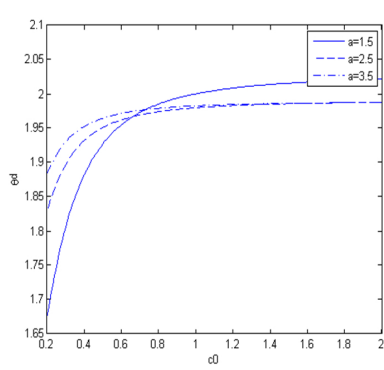

(b)
FIG. 5. Variations of $\varepsilon_{d}$ and $\theta_{d}$ with $c_{0}$ on the receiving plane at the propagation distance $\mathrm{z}=10 \mathrm{~km}$ under different $\alpha$ (a) $\sigma=1$ $\mathrm{cm}, \delta_{y y}=0.5 \delta_{x y}$, (b) $\sigma=1 \mathrm{~cm}, \delta_{y y}=0.5 \delta_{x y}$. Other parameters are same with Fig. 2.

\section{DISCUSSION}

(1) Both DOP and SOP fluctuate at the range of 0.01 $\mathrm{km}<\mathrm{Z}<1 \mathrm{~km}$ when propagating in oceanic turbulence and will reconstruct when $\mathrm{z}>1 \mathrm{~km}$. DOP and SOP get more fluctuations in weak turbulence and get larger self-reconstructed distance than that in strong turbulence.

(2) $S_{2}$ and $S_{3}$ in ocean waters fluctuate less than $S_{1}$ during the propagation but they will all reach the initial value if the propagation distance is long enough.

(3) DOP varies with more complexity with distance in ocean waters than SOP. The self-reconstructed distance of both decreases with the increase of $\chi_{t}$ and $\mathrm{w}$, but increases with the increases of $\xi$.

(4) When $\delta_{y y}=\delta_{x y}, P_{d}$ reaches the minimum value, and it's almost equivalent for different $\alpha$, but if $\delta_{x x \neq} \delta_{x y}$, as what have shown in Fig. 4(b), $P_{d}$ will get to minimum value at different $c_{0}$ for different $\alpha$.

(5) $\varepsilon_{\mathrm{d}}$ and $\theta_{\mathrm{d}}$ increase as increases but will change slowly when $c_{0}>1$. It means when other parameters are kept fixed, the coherence width $\delta_{x x}$ should be controlled to be smaller than $\delta_{x y}$ in order to obtain better SOP self-reconstructed properties, but these two 
Polarization Properties of Quasi-Homogeneous Beams Propagating in Oceanic Turbulence - Feinan Chen et al. 135

variables of SOP have no minimum values with the variation of $c_{0}$.

\section{CONCLUSION}

According to our analysis, the normalized Stokes parameters will be affected by temperature and salinity fluctuations so the DOP and SOP will also be affected. And $s_{1}$ fluctuates much more than $s_{3}$ and $s_{3}$, DOP varies with more complexity than SOP. Furthermore, both of normalized generalized Stokes parameters of QH beams have proved to be equal to the initial value if the propagation distance is long enough, so the self-reconstructed properties of DOP and SOP of such beams can also be proved. The choice of coherence width of the $\mathrm{QH}$ beams can help us to get the best self-reconstructed ability of DOP, but for two parameters of SOP, there are no best choices of these parameters. Our work may be helpful to develop underwater optical imaging or communication system.

\section{ACKNOWLEDGMENT}

The work is supported by National Natural Science Foundations of China (Grant No.61077012, 61107011).

\section{REFERENCES}

1. L. Mandel and E. Wolf, Optical Coherence and Quantum Optics (Cambridge University Press, Cambridge, UK, 1995).

2. O. Korotkova, B. G. Hoover, V. L. Gamiz, and E. Wolf, "Coherence and polarization properties of far fields generated by quasi-homogeneous planar electromagnetic sources," J. Opt. Soc. Am. A 22, 2547-2556 (2005).

3. Y. Xin, Y. Chen, and Q. Zhao, "Changes of spectrum of light scattering on quasi-homogenous random media," Proc. SPIE 6786, 67864S-1 67864S-9 (2007).

4. J. Chen, F. Chen, and Y. Chen, "Coherence properties of the scattered electromagnetic field generated by anisotropic quasi-homogeneous media," Opt. Commun. 285, 3955-3960 (2012).

5. J. Li, Z. Shi, and H. Ren, "Polarization modulation of electromagnetic waves scattered from a quasi-homogeneous anisotropic medium," IOP Science, 125705-1 125705-6 (2012).

6. K. Kwon, T. Son, C. Yeo, and B. Jung, "Numerical modeling of compression-controlled low-level laser probe for increasing photo density in soft tissue," J. Opt. Soc. Korea 15, 321-328 (2011).

7. Z.-f. Shu, X.-k. Dou, H.-y. Xia, D. Sun, Y. Han, H. Cha, D. Kim, G.-c. Wang, S. Baik, and D.-d. Hu, "Low stratospheric wind measurement using mobile rayleigh doppler wind LIDAR,” J. Opt. Soc. Korea 16, 141-144 (2012).

8. J. H. Lee, Y.-S. Jung, S.-Y. Ryoo, Y.-J. Kim, B.-U. Park, H.-J. Kim, S.-K. Youn, K.-W. Park, and H. B. Lee, "Imaging performance analysis of an EO/IR dual band airborne camera," J. Opt. Soc. Korea 15, 174-181 (2011).

9. W. Gao, "Changes of polarization of light beams on propagation through tissue," Opt. Commun. 260, 749-754 (2006).

10. W. Gao and O. Korotokova, "Changes in the state of polarization of a random electromagnetic beam propagating through tissue," Opt. Commun. 270, 474-478 (2007).

11. J. C. Ricklin and F. M. Davidson, "Atmospheric turbulence effects on a partially coherent Gaussian beam: implications for free-space laser communication," J. Opt. Soc. Am. A 19, 1794-1802 (2002).

12. M. Salem, O. Korotkova, and A. Dogariu, "Polarization changes in partially coherent electromagnetic beams propagating through turbulent atmosphere," Waves Random Media 14, 513-523 (2004).

13. H. Roychowdhury and E. Wolf, "Invariance of spectrum of light generated by a class of quasi-homogenous sources on propagation through turbulence," Opt. Commun. 241, 11-15 (2004).

14. F. D. Kashani, M. Alavinejad, and B. Ghafary, "Polarization characteristics of aberrated partially coherent flat-topped beam propagating through turbulent atmosphere," Opt. Commun. 282, 4029-4034 (2009).

15. X. Du, D. Zhao, and O. Korotkva, "Changes in the statistical properties of stochastic anisotropic electromagnetic beams on propagation in the turbulence," Opt. Express 25, 16913-16915 (2007).

16. Y. Zhou, K. Huang, and D. Zhao, "Changes in the statistical properties of stochastic anisotropic electromagnetic beams propagating through the oceanic turbulence," Appl. Phys. B 109, 289-294 (2012).

17. O. Korotkova and N. Farwell, "Effect of oceanic turbulence on polarization of stochastic beams," Opt. Commun. 284, 1740-1746 (2011).

18. W. Lu, L. Liu, and J. Sun, "Influence of temperature and salinity fluctuations on propagation behavior of partially coherent beams in oceanic turbulence," J. Opt. A: Pure Appl. Opt. 8, 1052-1058 (2006).

19. O. Korotkova, M. Salem, A. Dogariu, and E. Wolf, "Beam conditions of radiation generated by an electromagnetic Gaussian Schell-model source,” Opt. Lett. 29, 1173-1175 (2004).

20. O. Korotkova and E. Wolf, "Generalized Stokes parameters of random electromagnetic beams," Opt. Lett. 30, 198-200 (2005).

21. J. Tervo, T. Setala, and A. Roueff, "Twp-point stokes parameters: interpretation and properties," Opt. Lett. 34, 3074-3076 (2009).

22. B. Kanseri and H. C. Kandpal, "Experimental determination of two-point stokes parameters for a partially coherent broadband light beam," Opt. Commun. 283, 4558-4562 (2010).

23. B. Kanseri, S. Rath, and H. C. Kandpal, "Direct determination of the generalized stokes parameters from the usual stokes parameters," Opt. Lett. 34, 719-721 (2009).

24. Y. Zhu and D. Zhao, "Generalized stokes parameters of a stochastic electromagnetic beam propagating through a paraxial ABCD optical system,” J. Opt. Soc. Am. A 25, 1944-1948 (2008).

25. X. Y. Du and D. M. Zhao, "Changes in generalized stokes parameters of stochastic electromagnetic beams on propagation through $\mathrm{ABCD}$ optical systems and in the turbulent atmosphere," Opt. Commun. 281, 5968-5972 (2008). 